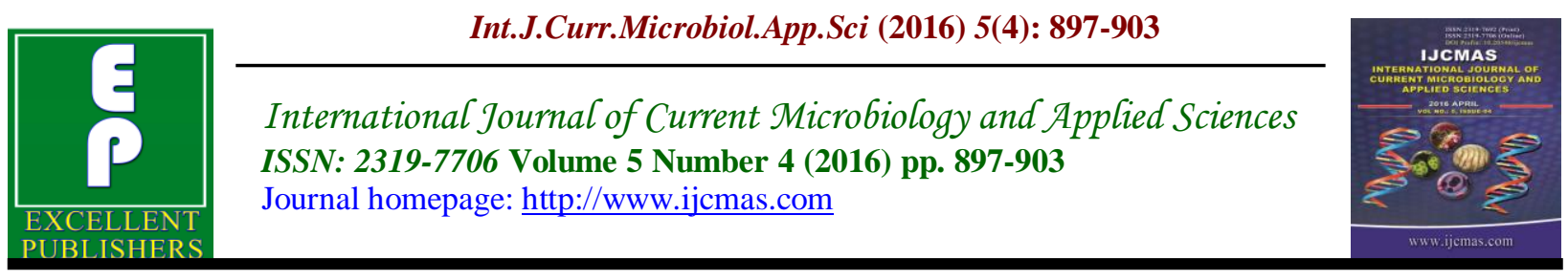

Original Research Article

http://dx.doi.org/10.20546/ijcmas.2016.504.101

\title{
Isolation of Urinary Tract Pathogens and Study of their Drug Susceptibility Patterns
}

\author{
Richa Mishra*, Deepti Saksena and Vinoy K. Shrivastava \\ Department of Biosciences, Barkatullah University, Bhopal, 462026 (M.P.), India \\ *Corresponding author
}

\begin{abstract}
A B S T R A C T
Keywords

Urinary Tract

Infections,

Drug Resistance,

Bacterial

Pathogens,

E. coli,

Antimicrobial

Susceptibility

Pattern.

Article Info

The objective of present study was to analyze urine specimen of patients suffering from UTI for the identification of pathogenic organisms and their drug susceptibility patterns. A total of 1255 (897 females and 358 males) patients with clinical symptoms of UTI were included in the study. Automated bioMérieux VITEK 2 compact bacterial identification and antibiotic susceptibility analyzer is used in the present study. A total of 1255 urine samples were collected and out of these 400 were growth positive isolates. Out of these isolates Escherichia coli $(42.5 \%)$ was found to be the most frequent causative agent of UTIs followed by Klebsiella spp (23.25\%), Pseudomonas spp. (12.5\%) and Enterobacter spp. (9.75\%), Candida spp. (5\%), Staphylococcus aureus (3\%), Acinetobacter spp. $(2.5 \%)$ and Proteus $(1.5 \%)$. All urinary isolates of E.coli including multidrug resistant strains were found to be highly sensitive for nitrofuratoin and pazufloxacin. Women are more prone towards UTI and E. coli is the main Accepted: 25 March 2016 Available Online: 10 April 2016 causative organism. The change in drug susceptibility pattern is notable since older drugs no longer show effectiveness, this is due to resistance developed by overuse or misuse of drugs. Comparative study should be repeated after regular intervals of time so that the changing patterns can be noted and choice of drug can be selected accordingly.
\end{abstract}

\section{Introduction}

In the clinical laboratory bacteria are cultured and precisely identified so that the appropriate medication can be selected. Gram negative bacteria are the most prevalent isolates from the UTI patients and urinary tract infection being commonest infection caused (Bashir et al., 2008; Mehta et al., 2012).

Microorganisms are not always harmful but when they are present in excess they become pathogenic and cause infectious diseases. Escherichia coli which is a natural inhabitant of gastrointestinal tract and urinary tract but when their number increases it becomes pathogen and causes Urinary Tract Infection (UTI).

In fact, Gram negative bacteremia is caused mainly due to UTI in patients of all ages and in elderly patients there is a high risk of morbidity and mortality. In recent years there has been an increase in bacterial UTI 
although several drugs are available and prescribed. This can be associated to the resistance developed by pathogens for antibiotics.

E. coli is the prominent causative uropathogen of UTI and among other pathogens of infection Pseudomonas spp., Candida spp., Klebsiella spp. and Enterobacter spp. are also found (Khoshhbakht et al., 2013). Resistance developed by microbes for antibiotics is a major problem which leads to improper treatment. An increase in the resistance for antibiotics is also because of haphazard use which is unchecked. Hence, antibiotics should be prescribed after urine culture \& sensitivity test (Hryniewicz et al., 2001).

In the present study emphasis has been given to the prevalence and susceptibility patters of uropathogens. The data analyzed gives basic knowledge for future study and can be useful for controlling UTI.

\section{Materials and Methods}

A total of 1255 (897 females and 358 males) patients with clinical symptoms of UTI were included in the study. Sterile disposable containers were used for collecting midstream urine samples $(4-5 \mathrm{ml})$ and transported immediately to laboratory. Urine routine examination was done using direct microscopy to count white blood cell (WBC). Cysteine lactose electrolyte deficient agar (CLED agar) plates were used for inoculation of urine samples by adopting Calibrated Loop Technique and kept for 24 hours incubation at $37^{\circ} \mathrm{C}$ aerobically.

Cultures without any colony at the end of 24 hours incubation were further incubated for 48 hours. Urine culture plates having significant growth were observed on the basis of colonial morphology and colony counts. A growth of $\geq 10^{5}$ colony forming units $/ \mathrm{ml}$ was considered as significant bacteriuria. Gram staining was performed to differentiate the Gram positive andGram negative organisms. Bacterial identification and antibiotic susceptibility was done by automated bioMérieux VITEK 2 compact analyzer as per Clinical and Laboratory Standards Institute (CLSI) guidelines.

The measure of a microorganism's susceptibility or resistance to an antibiotic was reported as the Minimum Inhibitory Concentration (MIC). E. coli (ATCC 25922), Staphylococcus aureus (ATCC 25923) and Pseudomonas aeruginosa (ATTC 27853) were used as quality control strains. The antibiotics tested were Amikacin, Gentamycin, Amoxicillin, Imipenem, Meropenem, Ertapenem, Cefoperazone, Cotrimaxozole, Pazufloxacin, Levofloxacin, Norfloxacin, Ciprofloxacin, Nitrofuratoin, (Piperacillin + Tazobactam) and (Ceftazidime + Clavulinic).

\section{Statistical Analysis}

Clinical data were entered in the worksheet of Statistical Package for Social Science (SPSS) software of version 21.0. Percentage and frequency were analyzed as descriptive findings.

\section{Results and Discussion}

A total of 1255 urine samples, $358(28.52 \%)$ were from males and $897(71.47 \%)$ were from females. Out of these samples 400 were growth positive isolates. Frequency of growth positive samples in males were $23 \%$ and the frequency of growth positive samples in females were $77 \%$. Frequency pattern of males and females were shown in Table 1

The present study findings showed that 
Escherichia coli (42.5\%) was found to be the most frequent causative agent of UTIs followed by Klebsiella spp (23.25\%), Pseodomonas spp. (12.5\%) and Enterobacter spp. (9.75\%),Candida spp. (5\%), Staphylococcus aureus (3\%), Acinetobacter spp. (2.5\%) and Proteus $(1.5 \%)$. The percentage of isolated uropathogens were shown in Table 2 and distribution of isolated uropathogens were shown in Fig 1.

High degree of resistance was observed to cefoperazone, ciprofloxacin, amoxicillin, cotrimaxozole whereas combination drugs (piperacillin + tazobactam), (ceftazidime + clavulinic) along with amikacin, gentamycin, ertapenam, meropenem, imipenem, levofloxacin, norfloxacin exhibit low degree of resistance. Although all urinary isolates of E.coli including multidrug resistant strains were found to be highly sensitive for nitrofuratoin and pazufloxacin.
E.coli and Klebsiella spp were highly sensitive to pazufloxacin, nitrofuratoin and imipenem and they were highly resistant to amoxicillin. Pseodomonas spp. was highly sensitive to (Piperacillin + Tazobactam) and pazufloxacin and it was highly resistant to amoxicillin. Percentage of antibiotic sensitivity pattern of most frequently isolated microorganisms were shown in Table 3 and distribution of antibiotic sensitivity pattern were shown in Fig 2.

Many researchers have observed that the cause of UTI and the drug susceptibility patterns of pathogens were found to be changing since recent years (Saleh et al., 2009; Shalini et al., 2011). In our study UTI is the most common bacterial infection caused by E.coli. It was also seen that females get UTIs more often than males(Ronald et al., 2009; Chaurasia et al., 2015).

Table.1 Incidence of Urinary Tract Infection according to Sex

\begin{tabular}{|c|c|c|c|c|}
\hline Sex & $\begin{array}{c}\text { No. of Samples } \\
\text { Studied }\end{array}$ & Frequency (\%) & $\begin{array}{c}\text { No. of Positive } \\
\text { Samples }\end{array}$ & Frequency (\%) \\
\hline Male & 358 & 28.52 & 92 & 23 \\
\hline Female & 897 & 71.47 & 308 & 77 \\
\hline Total & 1255 & 100 & 400 & 100 \\
\hline
\end{tabular}

Table.2 Number of Isolated Uropathogens

\begin{tabular}{|l|l|}
\hline Isolated Uropathogens & Number \\
\hline Escherichia coli & $170(42.5 \%)$ \\
\hline Klebsiella spp. & $93(23.25 \%)$ \\
\hline Pseodomonas spp & $50(12.5 \%)$ \\
\hline Enterobacter spp. & $39(9.75 \%)$ \\
\hline Candida spp. & $20(5 \%)$ \\
\hline Staphylococcus aureus & $12(3 \%)$ \\
\hline Acinetobacter spp. & $10(2.5 \%)$ \\
\hline Proteus & $6(1.5 \%)$ \\
\hline
\end{tabular}


Table.3 Percentage of Antibiotic Sensitivity Patterns of Most Frequently Isolated Microorganisms

\begin{tabular}{|c|c|c|c|c|}
\hline Class of Antibiotics & Antibiotics & \begin{tabular}{|l|}
$\begin{array}{l}\text { E.coli } \\
(\mathrm{n}=170)\end{array}$ \\
\end{tabular} & $\begin{array}{l}\text { Klebsiella } \\
(\mathrm{n}=93)\end{array}$ & $\begin{array}{l}\text { Pseudomonas } \\
(\mathbf{n}=50)\end{array}$ \\
\hline \multirow[t]{2}{*}{ Aminoglycosides } & Amikacin & 75.62 & 75.03 & 76.58 \\
\hline & Gentamycin & 64.43 & 61.84 & 63.08 \\
\hline Aminopenicillin & Amoxicillin & 14.24 & 19.05 & 10.13 \\
\hline \multirow[t]{3}{*}{ Carbapenems } & Imipenem & 86.55 & 80.34 & 85.83 \\
\hline & Meropenem & 83.66 & 78.48 & 81.53 \\
\hline & Ertapenem & 81.96 & 79.98 & 79.01 \\
\hline Cepphalosporins & Cefoperazone & 38.83 & 40.83 & 34.13 \\
\hline $\begin{array}{l}\text { Trimethoprim } \\
\text { Sulphamethoxazole }\end{array}$ & Cotrimaxozole & 35.60 & 38.96 & 29.38 \\
\hline \multirow[t]{4}{*}{ Quinolones } & Pazufloxacin & 94.62 & 81.28 & 90.05 \\
\hline & Levofloxacin & 68.14 & 65.73 & 60.34 \\
\hline & Norfloxacin & 71.27 & 68.23 & 62.89 \\
\hline & Ciprofloxacin & 41.53 & 44.03 & 23.53 \\
\hline Nitrofurans & Nitrofuratoin & 87.98 & 74.58 & 18.97 \\
\hline \multirow[t]{2}{*}{ Combinations } & $\begin{array}{ll}\text { (Piperacillin } & + \\
\text { Tazobactam) } & \end{array}$ & 78.27 & 72.09 & 92.07 \\
\hline & $\begin{array}{ll}\text { (Ceftazidime } & + \\
\text { Clavulinic) } & \end{array}$ & 77.61 & 71.53 & 75.93 \\
\hline
\end{tabular}

Fig.1 Showing Distribution of Isolated Uropathogens

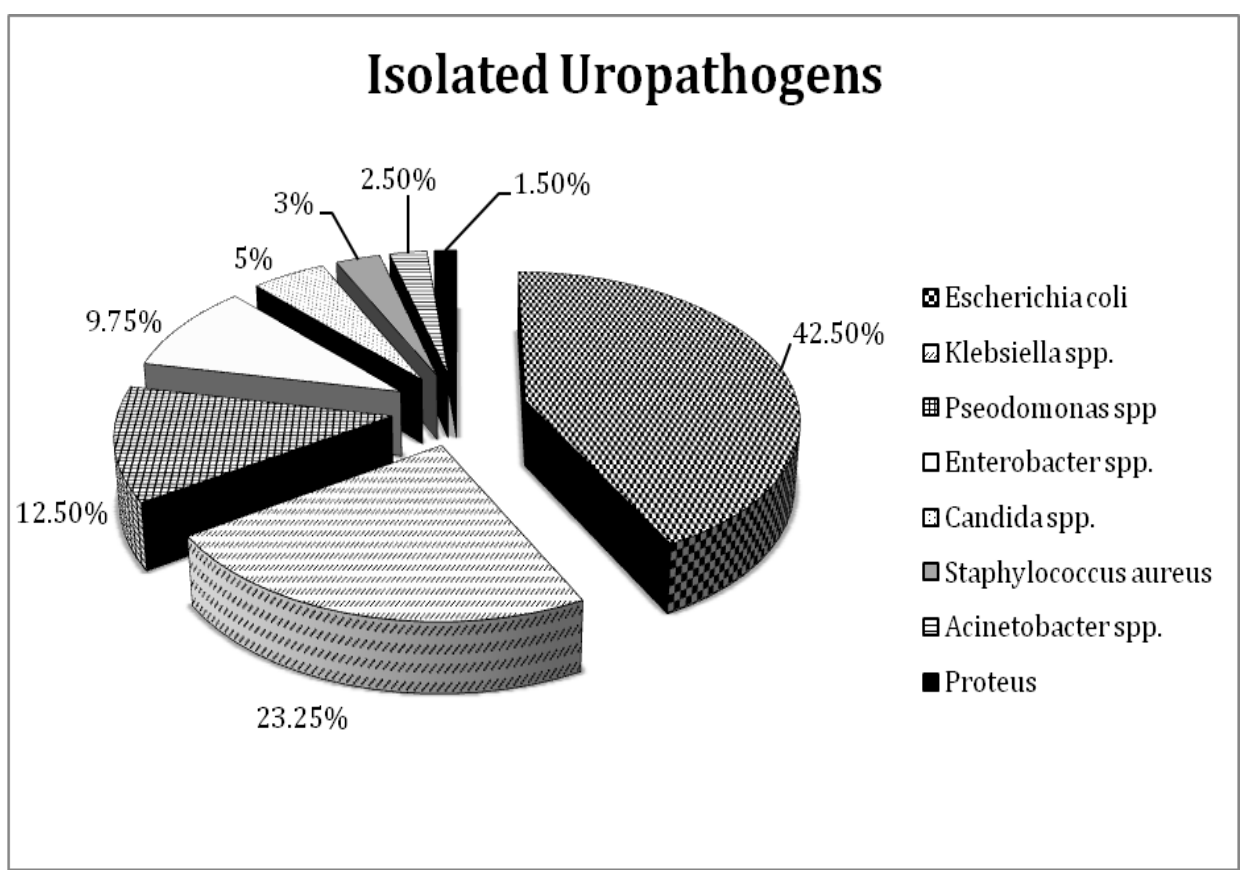


Fig.2 Showing Distribution of Antibiotic Sensitivity Patterns of Most Frequently Isolated Microorganisms

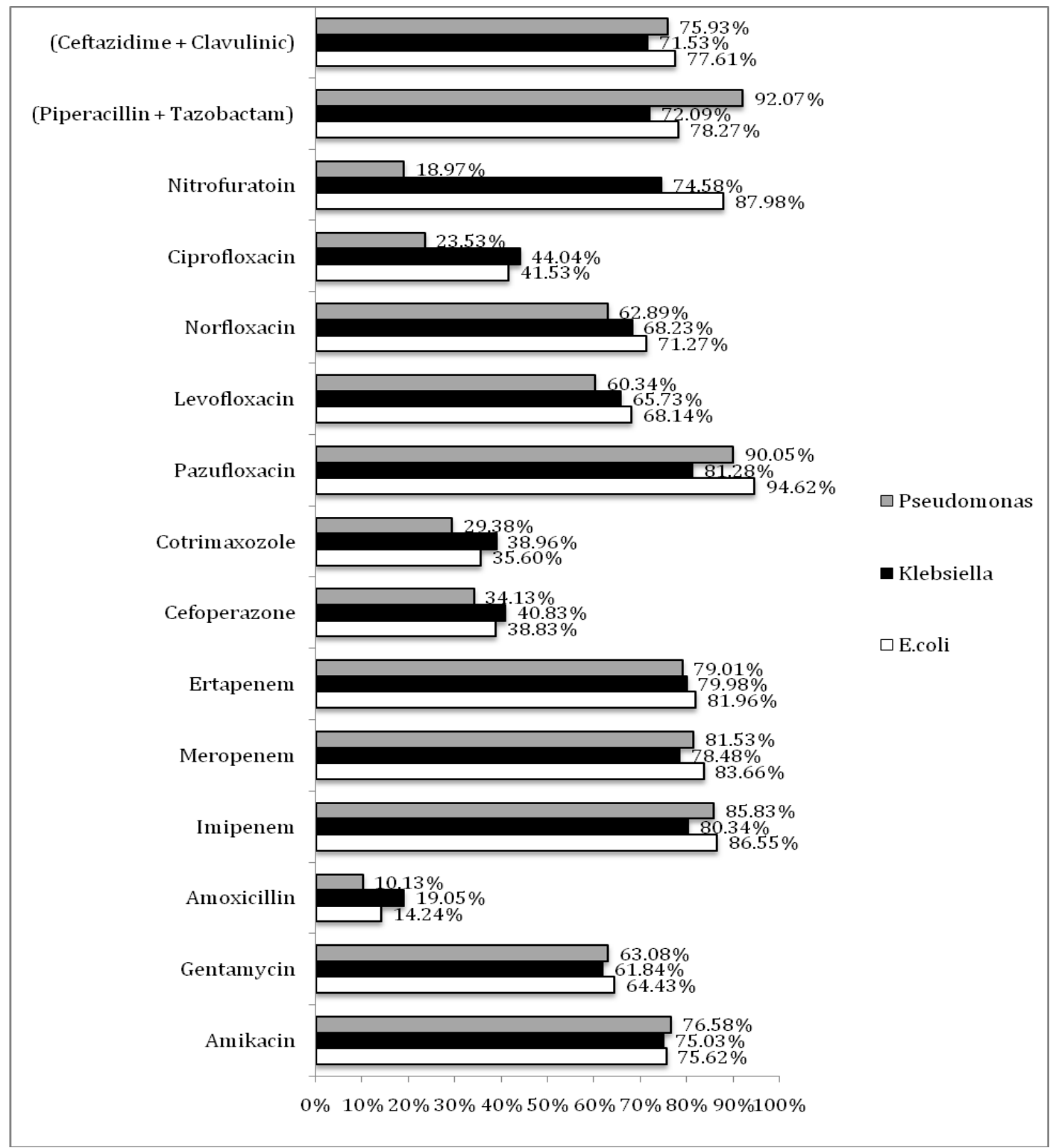

The most common organisms isolated in these patients were Escherichia coli(42.5\%), Klebsiella spp.(23.25\%), Pseodomonas spp. (12.5\%) and Enterobacter spp. (9.75\%), Candida spp. (5\%), Staphylococcus aureus (3\%), Acinetobacter spp. (2.5\%) and Proteus $(1.5 \%)$.All the micro-organisms obtained during this study were of clinical significance and were similar to the data recorded by (Hryniewicz et al., 2001).
Our study indicate that E.coli and Klebsiella spp were highly sensitive to pazufloxacin, nitrofuratoin and imipenem. Same has been recommended by (Shamataj and Vishwanath, 2012). Pseodomonas spp. washighly sensitive to (Piperacillin + Tazobactam) and pazufloxacin. (Niranjanand Malini, 2014) study is very much closely related to our study. 
The pathogen showed least sensitivity towards generally used antibiotics example amoxicillin etc. The present finding demonstrate that increased resistance in the amoxicillin against $E$. coli may reflect the overuse of aminopenicillin for treatment of UTI. Cefoperazone, ciprofloxacin, amoxicillin and cotrimaxozole are loosing effectiveness which is alarming because these antibiotics have been one of the best options for treatment of UTI.

In conclusion, Women are more prone towards UTI and among uropathogens Gram negative bacteria were most commonly isolated. E.coli was prominent among these. Broad-spectrum drugs can change normal microbial content of body (normal flora) which leads to secondary infection by multi drug resistant organism. Hence susceptibility patterns should be studied for the pathogens before prescribing antibiotics so that development of resistance against antibiotics can be checked.

The change in drug susceptibility pattern is notable since older drugs no longer show effectiveness, this is due to resistance developed by overuse or misuse of drugs.

The present study proves to be beneficial as it helps to identify the changing trends in the pattern of drug susceptibility. Hence, comparative study should be repeated after regular intervals of time so that the changing patterns can be noted and choice of drug can be selected accordingly. Persons with previous other infections or compromised immune system are much prone to UTIs hence, the frequency of occurrence of UTI is also responsible for the resistance developed for antibiotics in pathogens.

\section{Acknowledgement}

I am thankful to Department of
Biotechnology, New Delhi for providing fellowship as research assistance under the scheme DBT-IPLS-BU.

\section{References}

Bashir MF, Qazi JI, Ahmad N and Riaz S. Diversity of urinary tract pathogens and drug resistant isolates of Escherichia coli in different ageand gender groups of Pakistanis. Trop J Pharm Res 2008;7(3):1025-1031.

Chaurasia D, Shrivastava RK, Shrivastava SK, Dubey D and Songra MC. Bacterial pathogens and their antimicrobial susceptibility pattern isolated from urinary tract infection in a tertiary care centre. International Journal of Pharmacy \& Biosciences 2015; 1 .

Hryniewicz K, Szczypa K, Sulikowska A, Jankowski K, Brtlejewska $\mathrm{K}$ and Hryniewicz W. Antibiotic susceptibility of bacterial strains isolated from urinary tract infections in Poland. J Antimicrob Chemother 2001;47:773-780.

Khoshhbakht R, Salimi A, Aski HS and Keshavarzi. Antibiotic susceptibility of bacterial strains isolated from urinary tract infections in Karaj.Jundishapur J Microbiol 2013;6(1):86-90.

Mehta M,Bhardwaj S and Sharma S. Prevalence and antibiotic susceptibility pattern of multi drug resistant Escherichia coli isolates from urinary tract infection patients. Int J Life SciPharma Res 2012;2:L6L11.

Niranjan V and Malini A. Antimicrobial resistance pattern in Escherichia coli causing urinary tract infection among inpatients. Indian J Med Res 2014;139:945-948. 
Ronald PO, Lizzie JHand Keith SK. Antibiotic resistance in urinary isolates of Escherichia coli from college women with urinary tract infections. Antimicrob Agents Chemother 2009;1285-1286.

Saleh AA, Ahmed SS, Ahmed M, Sattar ANI and Miah MRA. Changing trends in uropathogens and their antimicrobial sensitivity pattern. Bangladesh J Med Microbiol2009;3(01):9-12.
Shalini, Joshi MC, Rashid MK and Joshi HS. Study of antibiotic sensitivity pattern in urinary tract infection at a tertiary hospital. Natl J Integr Res Med 2011;2:43-46.

Shamataj KR and Vishwanath G. Bacteriology of urinary tract infection and antibiotic susceptibility pattern in a tertiary care hospital in South India. Int $\mathbf{J}$ of Medical Science and Public Health 2012;109-112.

\section{How to cite this article:}

Richa Mishra, Deepti Saksena and Vinoy K. Shrivastava. 2016. Isolation of Urinary Tract Pathogens and Study of their Drug Susceptibility Patterns. Int.J.Curr.Microbiol.App.Sci. 5(4): 897-903. doi: http://dx.doi.org/10.20546/ijcmas.2016.504.101 